\title{
Los usos detransitivizadores del clítico se en español: construcciones media espontánea o decausativa, pasiva e impersonal
}

\author{
Adriana Zurlo
}

UNNE-CONICET

\section{Resumen}

En el artículo explicamos, desde la combinación de un enfoque tipológico-funcional (Givón, 1984; Bogard, 2008; Mendikoetxea, 1999) y estructural (Barrenechea-Rossetti, 1984), las construcciones del español en las que ocurre el clítico se junto a un único argumento nominal y un verbo intransitivo, es decir, construcciones que tradicionalmente han sido agrupadas bajo el nombre de pasiva refleja e impersonal.

Palabras claves: construcciones con clítico se, semántica, tipología funcional.

\section{Introducción}

Un tópico recurrente en las gramáticas del español es la difícil explicación de la correlación forma/funciones del clítico se en estructuras verbales formalmente similares y funcionalmente divergentes como las que se presentan en los enunciados (1) a (6). Si intentamos diferenciarlas por su significado, vemos que: (1) tiene valor de evento espontáneo, (2) accidental, anticausativo o también llamada decausativa; (3) pasiva; (4 a 6) impersonal omisión o indeterminación del agente-. La mayoría de estas construcciones (excepto 1) comparte la idea de una inducción externa -humana/no humana- esquemática.

(1) La luz se prendió sola.

(2) La planta se quebró (con el viento).

(3) Muchos libros se donaron.

(4) Se solicitan arquitectos.

(5) Se aprobó la ley de Bosques.

(6) Se eligió a los finalistas.

En este trabajo nos proponemos analizar construcciones verbales con un clítico etimológicamente reflexivo de tercera persona como las de los ejemplos (1) a (6) que, tradicionalmente, han recibido el nombre de pasiva con se e impersonal. Compararemos estos tipos de estructuras intentando diferenciarlas a partir de sus rasgos semánticos, morfosintácticos y pragmáticos. Algunos de los interrogantes que pretendemos respondernos en el trascurso del trabajo son:

a) ¿Cuáles son los rasgos formales/funcionales específicos de cada estructura?

b) ¿Es posible explicar esa semejanza formal, considerando el clítico se como marcador detransitivizador, como operación que permite codificar la reorganización o eliminación o de argumentos sintácticos centrales $(\mathrm{A}, \mathrm{S}, \mathrm{O})$ ?

Para respondernos, complementaremos dos explicaciones teóricas: estructuralismo (Barrenechea y Rosetti, 1984, 1979) y tipología funcional (Givón, 1984; Payne, 1997; Creissels, 2006) y las aplicaremos en el análisis y explicación de los enunciados registrados en nuestro corpus. 
Teniendo en cuenta las premisas teórico-metodológicas, en nuestro análisis observaremos los siguientes aspectos:

- La concordancia sintagma nominal-verbo;

- La clase de verbo (aspecto lexical y morfológico); tipo de verbo (estado-evento, acción)

- La presencia y características morfosintácticas y semánticas del argumento sujeto;

- La presencia o no de objeto (directo) y sus características semántico-sintácticas;

- La función/ significación del clítico se.

$\mathrm{Si}$ bien, muchos otros autores, desde diversas corrientes teóricas, han analizado construcciones como las de (1) a (6), en las que el clítico se ocurre antepuesto a un verbo intransitivo y junto a un único argumento nominal (por ejemplo: Mendikoetxea, 1999; Maldonado, 1999; Bogard, 2008) creemos que el valor de esta explicación reside en el intento de complementar dos perspectivas teóricas para comprender la complejidad de estas estructuras.

\section{La explicación estructural de las construcciones verbales pronominales}

Desde la linguística estructural (Barrenechea y Rosetti, 1984, 1979), la pasiva con se ha sido definida por oposición a la pasiva perifrástica y a las impersonales; pasiva con se e impersonales pertenecen al grupo de las cuasi-reflejas-.

La pasiva refleja se diferencia de la perifrástica por la ausencia de verbo pasivo (ser + participio pasado) y de complemento agente; aunque lo último no es exclusivo de la perifrástica.

Respecto de las impersonales, la pasiva refleja se diferencia por el comportamiento del sintagma nominal que ocurre junto al verbo, el cual, en general, es concordante y, por tanto, puede ser considerado sujeto. Este comportamiento, sin embargo, es fluctuante y con ello, el criterio de distinción se muestra insuficiente como lo observan las autoras a partir de un estudio experimental sobre los usos de las dos formas de voz pasiva en el habla de Buenos Aires (1979).

El sistema en el cual estaría integrada y definida la pasiva refleja, sería el que figura en la tabla 1, en la cual resumimos las diferencias morfosintácticas percibidas por Rosetti, junto a los ejemplos respectivos.

\begin{tabular}{|c|c|c|c|c|c|}
\hline & \multicolumn{5}{|c|}{$\begin{array}{c}\text { CONSTRUCCIONES ENDOCÉNTRICAS VERBALES PRONOMINALES } \\
\text { DE PERSONA COINCIDENTE }\end{array}$} \\
\hline \multirow{4}{*}{ RASGOS } & \multirow[t]{2}{*}{ REFLEXIVA } & \multirow[t]{2}{*}{ RECIPROCA } & \multicolumn{3}{|c|}{ CUASI-REFLEJAS } \\
\hline & & & \begin{tabular}{|l|} 
DE TODA \\
PERSONA \\
\end{tabular} & $\begin{array}{l}\text { DE 32 P. (Con } \\
\text { S) }\end{array}$ & $\begin{array}{l}\text { DE 3a P. (Con } \\
\text { S) }\end{array}$ \\
\hline & $\begin{array}{l}\text { Concordancia } \\
\text { S-V-OD. }\end{array}$ & $\begin{array}{l}\text { Concordancia } \\
\text { S-V-OD }\end{array}$ & $\begin{array}{l}\text { Concordancia } \\
\text { S-V-OD. }\end{array}$ & $\begin{array}{l}\text { Concordancia } \\
\text { S-V-OD }\end{array}$ & $\begin{array}{l}\text {-Sin } \\
\text { concordancia } \\
\text { - Verbo en } \\
\text { singular. }\end{array}$ \\
\hline & $S E=\mathrm{OD}$ & $S E=\mathrm{OD}$ & $S E \neq \mathrm{OD} / \mathrm{OI}$ & $S E \neq \mathrm{OD} / \mathrm{OI}$ & $S E \neq \mathrm{OD} / \mathrm{OI}$ \\
\hline
\end{tabular}




\begin{tabular}{|l|l|l|l|l|l|}
\hline & $\begin{array}{l}\text { Admite } \\
\text { refuerzo } \\
\text { reflexivo (a si } \\
\text { mismo, etc.) }\end{array}$ & $\begin{array}{l}\text { Admite } \\
\text { circunstancial } \\
\text { intensificador } \\
\text { (entre si, } \\
\text { mutuamente) }\end{array}$ & $\begin{array}{l}\text { No admite } \\
\text { refuerzo } \\
\text { reflexivo ni } \\
\text { circunstancial } \\
\text { intensificador }\end{array}$ & $\begin{array}{l}\text { No admite } \\
\text { refuerzos }\end{array}$ & $\begin{array}{l}\text { No admite } \\
\text { refuerzos }\end{array}$ \\
\hline EJEMPLOS & $\begin{array}{l}\text { Inés se mira } \\
\text { en el espejo. }\end{array}$ & $\begin{array}{l}\text { Todos sedos se } \\
\text { saludan. }\end{array}$ & $\begin{array}{l}\text { Se venden } \\
\text { marcharon. }\end{array}$ & $\begin{array}{l}\text { Se escucha a } \\
\text { todos. } \\
\text { Se vive bien. }\end{array}$ \\
\hline
\end{tabular}

TABLA 1. CONSTRUCCIONES ENDOCENTRICAS VERBALES PRONOMINALES.- (Rosetti, 1984)

Si bien, esta perspectiva analiza el comportamiento del clítico, aplicando rigurosamente los criterios formales (por ejemplo, la correferencialidad que establece el clítico con algún otro sintagma nominal (Sujeto/Objeto-); la concordancia nominal-verbal) estos criterios, estrictamente sintácticos, se muestran insuficientes para comprender las sutiles diferencias semántico-pragmáticas que el uso de estas estructuras acarrea, por ello, esta explicación no puede dar cuenta de los matices semánticos (de aspecto, de topicalidad) que el clítico aporta.

\section{EI aporte de la lingúistica tipológica y funcional}

La perspectiva tipológica focaliza los fenómenos linguísticos partiendo de la comparación translinguística y de una serie de postulados, fundamentalmente, el de la posibilidad de hallar determinados universales linguísticos; por ejemplo, la transitividad entendida como la relación entre un agente (A) y un paciente (P/O), los sistemas de alineación de los argumentos S, A y P, las operaciones de reajuste de la transitividad o reorganización de los participantes en las cláusulas.

Por otra parte, la linguística funcional parte de la premisa de la motivación semánticopragmática de toda forma. Es decir, la forma del mensaje siempre responde a una intencionalidad y se origina en un contexto comunicativo. La necesidad del hablante de hacerse comprender lo lleva a elegir, de entre las posibilidades que su lengua le ofrece, la estructura más adecuada para expresar sus conceptualizaciones acerca del mundo que lo rodea. Dicho de otro modo, mediante determinados procedimientos o mecanismos lingulistico-cognoscitivos podemos conceptualizar eventos y situaciones focalizando ciertos participantes o aspectos involucrados en aquellos. Estas estrategias universales han sido denominadas, desde diferentes enfoques, operaciones de ajuste de valencia, voz o incluso diátesis ${ }^{77}$.

De acuerdo con las líneas teóricas mencionadas observaremos el conjunto de las construcciones con clítico se, considerándolas como mecanismos de ajuste de valencia, que además de promover o eliminar argumentos sintácticos centrales (Sujeto, Agente y Objeto -P-)

" Cfr. Payne, (1997) quien habla de operaciones de ajuste de valencia. Por otro lado, García Miguel utiliza el término de diátesis (García Miguel; 2001 a: 3-4) para indicar precisamente las variaciones en la transitividad de la cláusula $y$ en la prominencia de cada participante; es decir, aquello que hemos denominado mecanismos de ajuste de valencia y, además, agrega: Las principales operaciones cognitivas implicadas tienen que ver con la selección del ámbito de predicación (qué entidades de las presentes en el estado, cosas son seleccionadas para su representación linguilística), con la relación perfilada por el predicado (y por tanto con la selección de los participantes centrales) y la orientación o selección del punto de partida en la conceptualización del evento" (García Miguel; 2001: 4). 
constituyen estrategias lingúísticas con determinados efectos semántico-pragmáticos: ocultar la identidad de un agente y, por lo tanto eliminar su responsabilidad (impersonal), destacar el grado de afectación o involucramiento de un participante en un evento o estado, focalizar en el cambio de estado resultante -pasiva, anticausativa-, etc.

Givón (1984:564-623) propone observar el dominio de la pasividad -detransitivizaciónen primer lugar, considerando que en toda lengua esta codificado por una familia de construcciones. Por otro lado, define la voz pasiva como un cambio de perspectiva, una operación sintáctica-pragmática por la cual (y a diferencia de la activa): -) A es eliminado de su prototípica posición de tópico ( $\mathrm{S}$ en cláusulas transitivas activas); b) el argumento no-A es promovido a la posición de tópico y, c) se estativiza (se hace intransitivo) el verbo. Este autor sugiere que la construcción en español, tradicionalmente llamada pasiva con se al igual que la impersonal constituyen dos tipos de pasiva (promocional y no promocional respectivamente) que podrían derivar diacrónicamente de una misma fuente, el clítico originariamente reflexivo del latin.

Por otra parte, Mendikoetxea (1999:1631-1722) ha planteado la existencia en el español de construcciones medias, pasivas e impersonales marcadas con el clítico se. La voz media puede ser definida semánticamente, de acuerdo con esta autora como una acción o proceso verbal que afecta al sujeto gramatical, que, con verbos transitivos se corresponde con el objeto nocional del verbo. Formalmente, se caracteriza por la presencia de afijos verbales de persona $o$ elementos pronominales (me, te, se, nos...) según Mendikoetxea (1999: 1653) y, siguiendo a (Bogard, 2008: 34) como constituyente verbal o cuasiafijo. Además, Mendikoetxea (1999:\$26) sugiere que entre las construcciones con clítico se podemos hallar: a) pasivas (Ayer se lavaron todas las camisas); b) impersonales (Se asustó a los niños) y c) medias. Entre estas ultimas hallamos subtipos de construcciones; medio-pasivas, oraciones de carácter genérico que predican una propiedad inherente del sujeto (Esta camisa se lava muy bien), medioimpersonales ( $A$ estos niños se les asusta fácilmente) e incoativas que denotan un cambio físico de estado o de posición conceptualizado como espontáneo, sin la intervención deliberada de un agente o causa (El bosque se quemó).

\section{Análisis de los datos} aspectos:

En este apartado analizaremos algunos enunciados, teniendo en cuenta los siguientes

a) Verbo -estructura léxico-semántica y aspecto morfológico-;

b) Frase nominal (caracteristicas morfosintácticas y semánticas del argumento sujeto/objeto);

c) Fuerza inductora (agentividad/causatividad);

d) La función/ significación del clítico se.

En los siguientes correlatos podemos observar un enunciado en el que ocurre el clítico se junto a verbos intransitivos que denotan un cambio de estado físico o de posición, en algunos casos espontáneo (7), accidental (8), en todos los casos, sin inducción externa definida ni mucho menos un agente; además, cuando el verbo lo permite, un enunciado transitivo activo, en el que se manifiesta la relación entre A (Agente) - Objeto (Paciente/Tema). 
(7) Se cayó la caja -solaPRET.PERF.s/3sg. S (T)/sg (CIRC.Modo) ${ }^{78}$

(7b) Se cayeron todas las cajas -solasPRET.PERF.s/3pl. S (T)/-pl (CIRC.Modo)

(8) -¿Quién tiró agua ahí?

- Nadie tiró. Se volcó, que es diferente.

PRET.PERF.ș/3sg

(9a) Se apagó sola la luz de la pieza.

PRET.PERF.s/3sg. CIRC S (T)/sg

(9b) Los vecinos apagaron

el fuego.

Activa. (TR) $S(A)$

PRET. PERF.s/3pl O (P)/sg

(10a) ¡Se rompió la fuente! (por el calor del horno) PRET.PERF.s/3sg. S (T)/sg

(10b) Juan rompió

la foto de su novia

S(A) PRET.PERF.s/3sg. O (T)/sg

Activa (TR)

(11) Se quebró el rosal (con el granizo)

PRET.PERF.s/3sg. S(T)/sg CIRC. (Cau)

(12) (Por la sequía) se perdió la cosecha.

CIRC (CAU) PRET. PERF. s/ 3sg. S (T)/sg.

(13) Se enfrió la comida ${ }^{*}$ con/por el ventilador). ${ }^{79}$

PRET.PERF.s/3sg. S (T)/sg CIRC. (Cau)

En los enunciados (7), (8), (9a) a (10a) encontramos verbos intransitivos que denotan cambio de estado físico (apagarse; romperse) o de posición (volcarse, caerse), es decir, verbos causativos de causa externa de acuerdo con Mendikoetxea (1999: § 25.2.1.). Estos eventos

${ }^{78}$ Las siglas y abreviaturas empleadas en el análisis morfosintáctico del español son las siguientes:

A: agente; CAU: Causa (circunstancia de); CIRC: circunstancial; DAT: dativo; FREC: frecuencia (circunstancial de); FV: Frase verbal; INSTR: Instrumento (circ. De ); LUG: Lugar (circ. De ); $Q$ : objeto; P: paciente; PL: plural; PPIO PDQ: participio pasado; PRET.IMPERF: Pretérito Imperfecto M. Indicativo; PRET.PERF.s: Pretérito Perfecto simple, m. Indicativo; PTE: Presente, M. Indicativo; S: Sujeto; SG: Singular, I: tema; Tpo: tiempo (circunstancia de);TR: transitiva; VA: voz activa; VP: voz pasiva; 3 sg: tercera persona sujeto, singular; 3 pl: tercera persona, sujeto, plural; 10 : Primera persona, Objeto.

${ }^{79}$ En el desarrollo del análisis el símbolo (*) indica que el enunciado es inaceptable o agramatical. 
ocurren espontáneamente, sin la intervención de un agente o de una causa. En algunos casos es posible reponer mediante un circunstancial u oblicuo de instrumento o causa la fuerza inductora del cambio de estado como en (10a, 11, 12), en otros caso es agramatical (13). El aspecto perfectivo de los verbos aporta una lectura puntual, télica, en la cual el cambio de estado puede ser conceptualizado como acabado, concluido.

Antepuesto al verbo ocurre el clítico se. Pospuesto aparece un sintagma nominal concordante $(7 \mathrm{a} / 7 \mathrm{~b})$ que cumple función de sujeto y cuyos rasgos semánticos lo aproximan a un paciente prototípico -ente inanimado, afectado por el cambio de estado, definido porque en la mayoría de los casos está determinado por artículo o adjetivos y conocido-.

En los próximos correlatos observaremos qué implicancias tiene las variaciones aspectuales y de los rasgos semánticos del argumento afectado en este tipo de construcciones.

En los correlatos de $(14,15)$ tenemos un verbo intransitivo de cambio de estado (tildarse, con su variante coloquial colgarse, también detenerse, pararse, descomponerse).

Se tildó

PRET.PERF. s. /3sg S(T)/sg.

(14b)
Se tildaba PRET.IMPERF./3sg S (T)/sg.
i computado

mi computadora.

odas las computadoras se tildaron

$\mathrm{S}(\mathrm{T}) / \mathrm{pl}$.

PRET. PERF. s/3pl.

(14d) "Se me

colgó

la PC. Aguantáme un cacho".

(DAT)/10sg PRET. PERF s./3sg. S(T)/sg.

(15a)

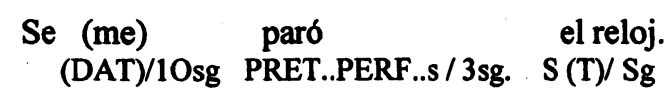

(15b) $\mathrm{Se}(\mathrm{me})$

paró

el reloj.

(DAT)/1Osg PRET. PERF. s/3sg. S (T)/Sg

$(* / ?$ con los golpes/las caídas)

(15c) El reloj

se paraba

a las tres.

S (T)/sg. PRET.PERF.s/3sg. CIRC. (Tp)

Si observamos el único sintagma nominal (14a, b, c, 14d) vemos que:

a) sus rasgos semánticos lo aproximarían a un paciente o tema, en tanto es no humano, inanimado, concreto (artefacto tecnológico), afectado. Al tratarse de un artefacto tecnológico es posible conceptualizar que el argumento afectado alcanza su estado por una autoinducción, energía interna indeterminada, es decir, es posible conceptualizar una causa interna.

$\mathrm{Si}$ atendemos al número, es una entidad singular $(14 \mathrm{a}, 14 \mathrm{~d})$, individualizada; la ocurrencia del posesivo (14a) lo convierte en un objeto definido y conocido. Obsérvese que la indeterminación del argumento vuelve inaceptable la construcción como en: 
14e) *Se tildó una/alguna computadora. $\quad / *$ Alguna computadora se tildó.

b) Sintácticamente cumple la función de sujeto porque concuerda en número con el verbo (14a y 14c); 2) ocupa una posición central ${ }^{80}$, adjunta al verbo: preverbal (14c); posverbal (14a) y, finalmente, 3) ningún otro argumento nominal posee mayor prominencia -no está representado el inductor-.

Por otro lado, si atendemos la estructura lexical y aspecto morfológico del verbo vemos que:

-) por su significado inherente, el verbo denota un cambio de estado no volitivo, que no trascurre en un tiempo extenso (también llamados logros al igual que por ejemplo; morirse, explotar, parar, romperse), puntual/ télico, sin focalizar inducción externa definida -no es un verbo que requiera agente-. Obsérvese que la inclusión de un inductor externo (causa o agente) se torna inaceptable:

(14e) Mi computadora se tildó (*con/por ese antivirus / el uso de Internet/ por Juan).

-) La marcación morfológica perfectiva -pretérito perfecto simple- permite conceptualizar el evento como acabado, ocurrido (aspecto télico): 14a, c, d. La marca imperfectiva (14b) introduce una lectura habitual o repetitiva, desfocalizando la porción terminal del cambio de estado y subrayando, en cambio, lo estable, homogéneo y/o repetitivo del evento. Nos dice, Maldonado (1999: 295) que un rasgo formal de esta construcción es la restricción aspectual, por la cual requiere la combinación predicado cambiante (aspecto lexical) y marca perfectiva (aspecto morfológico) para focalizar el cambio de estado como evento global, acabado; aunque este autor denomine la construcción con la etiqueta de media de prominencia terminal.

Sintáctica y semánticamente, es una construcción próxima a las absolutas-intransitivas, con único participante afectado en función sujeto y perfectividad morfológica ${ }^{81}$.

En el siguiente correlato (16) podemos observar otro tipo de construcción: pasiva promocional, en la que, al parecer, se focaliza el resultado de la acción y al participante afectado/efectuado. Este último promovido a la función sujeto.

(16a) Se construyeron casas baratas

(Barrenechea-Rosetti, 1979:

67)

$$
\text { PRET. PERF.s./3pl O (T)/pl. }
$$

(16b) Esa empresa construye casas más baratas. A/sg. PTE/3sg O (T)/pl.

(Activa. TR)

\footnotetext{
80 La posición que ocupa corresponde generalmente en espaniol (dado que el orden de los constituyentes es relativamente libre en esta lengua) a la del sujeto en oraciones intransitivas activas o a la del objeto en transitivas activas.

81 La perfectividad morfológica es otro rasgo importante en estas construcciones puesto que la variación temporoaspectual, brinda una visión procesual del evento, focalizándolo como cambio habitual, natural de un ente (Las paredes se rajan (por las vibraciones); los metales se dilatan con el calor), o aportando una lectura iterativa: Se tildaba mi computadora cada vez que la prendía).
} 

pasiva)

(16d) Se construye casas más baratas en el sur. / Se construye casas baratas.

En relación a las construcciones anteriores, observamos que:

a) según su estructura léxico-semántica, este verbo denota una actividad que se desarrolla en el tiempo -durativa- y requiere un agente prototípico (+humano; +control; +volición) que no está expresado. La perfectividad morfológica aporta una lectura terminal, global del evento (16a), en oposición al aspecto imperfectivo que impone una lectura habitual (17a/b; 18).

b) La frase nominal concuerda con el verbo. Es posverbal y puede ser remplazada por pronombre objetivo (las) -operación que exige ajustar el número verbal-. Semánticamente, se trata de un ente concreto, inanimado, afectado (paciente o tema). No presenta determinación deíctica -no es una instancia, como en (14a)- pero es definido mediante el adjetivo o construcción calificativa (16a, 17a).

c) Si bien no está expresado o nominalizado el agente, la mayoría de los verbos lo tienen lexicalizado en su estructura: construir, oir, decir, dictar, vender denotan situaciones transitivas prototípicas que involucran agentes humanos. La impersonalidad estaría dada porque el significado predominante es el de un agente indeterminado o genérico: uno, alguien, un grupo humano.

(17a) Se oyen extraños ruidos. $\mathrm{PTE} / 3 \mathrm{pl} \mathbf{O}(\mathrm{T}) / \mathrm{pl}$.

(17b) Se oye el ruido de los motores y la bocina de los coches PTE/3sg $\mathrm{O}(\mathrm{T})$.

(Barrenechea-Rosetti, 1979: 63)

(18) En la calle se veía poca gente.

IIRC. PRET.IMPERF/3sg O (P)/sg.

63)

El correlato (19a, b y c) nos permitirá observar y explicar el comportamiento de la concordancia sintagma nominal-verbo.

(19a) Se alquilan habitaciones PTE/3pl O(T)/pl.

(Barrenechea-Rosetti, 1979: 70)

(19b) Se alquila habitaciones. / Se las alquila PTE/3sg O (T)/pl.

(Barrenechea-Rosetti, 1979: 70)

(19c) Se alquila este local. PTE/3sg $O(T) / s g$.

(Cartel en la vía publica)

(20a) Se utilizan poco esos reglamentos PTE/3pl CIRC $O(T) / p l$.

(Barrenechea-Rosetti, 1979: 69) 
(20b) Se utiliza las visitas de otras personas. PTE/3sg. $\mathrm{O}(\mathrm{T}) / \mathrm{pl}$.

(Barrenechea-Rosetti, 1979: 63)

En primer lugar, la agentividad, no nominalizada pero lexicalizada en el verbo, permite distinguir en estas estructuras, un agente humano indeterminado (A) de un argumento afectado (Objeto-Tema/Paciente). Nos dice Maldonado (1999: 328) que con verbos de requisito alto de agentividad, hay un agente esquemático con función sujetiva y (...) las anteposiciones del tema constituyen un movimiento hacia una posición de tópico. Es decir, este tipo de estructuras se caracterizaría por la presencia implícita o sobreentendida de un agente -lexicalizado en el verbo- y un participante afectado/efectuado $(O)$ discursivamente prominente. En este sentido, la concordancia del único sintagma nominal con el verbo constituye una estrategia para focalizar el objeto afectado o efectuado, promocionándolo al status de tópico, pero sin que pierda necesariamente su función objetiva (obsérvese que los ejemplos 19a y 19c permiten ambas lecturas pasiva e impersonal). La falta de concordancia permitiria remarcar la prominencia del argumento agentivo frente al afectado, especialmente, cuando el participante afectado también es humano ${ }^{82}$; por ello, además de posponerse el sintagma nominal -posición típica del objetose lo codifica con marca de acusativo o preposición de objeto (21 a 24a).

Esta última distinción semántica, ya ha sido realizada por Barrenechea-Rosetti (1979: 70), quienes la han formulado en términos de persona/no persona. Así, los sintagmas nominales cuyos referentes son personas (21 a 23) están encabezados por una preposición $a$, es decir, con la marca del objeto directo (acusativo). Como mencionábamos anteriormente, estos verbos demandan un agente prototipico humano indeterminado; ahora bien, cuando otro argumento + humano compite en prominencia (paciente, dativo, receptor o benefactivo), la concordancia y la marcación acusativa indican el modo de leer el evento -conceptualizado como situación transitiva- y, por ello, constituyen estrategias para destacar al participante agentivo, el cual puede ser sobreentendido o restituido a pesar de no estar presente en el enunciado.

Se adiestra a las voluntarias.

PTE/3sg O (P/DAT)/pl. (+ Humano)

(Barrenechea-Rosetti, 1979: 69)

Se amonestó a los alumnos. PRET PERF.s./3sg O (DAT/REC)/ pl. (+ Humano)

$$
\begin{array}{ll}
\text { Se tomó } & \text { a muchos peones. } \\
\text { PRET. PERF.s./3sg } & \text { O (DAT /pl. (+ Humano) }
\end{array}
$$

(Barrenechea-Rosetti, 1979: 63)

Cabe recordar que, como lo sugiere Givón (1984:566-600), es posible distinguir dos subtipos de pasivas o mejor dicho de construcciones detransitivas en las cuales no se focaliza al agente o inductor del evento: por un lado, la pasiva promocional (pasiva en la cual el argumento

\footnotetext{
${ }^{82}$ El español, en la voz activa, muestra en la marcación de sus argumentos sintácticos preferencia por el caso o rol semántico de agente y, secundariamente dativo, paciente etc. (Ver escalas de agentividad y topicalidad en Givón, 1984: 89 y ss.)
} 
afectado -P/O- establece concordancia con el verbo) y la impersonal o pasiva no promocional, en la cual el participante afectado compite en topicalidad con el agente pero conserva su marca de acusativo; esta último estructura se observa en los ejemplos en los mencionamos la presencia de un agente lexicalizado en la base verbal y un sintagma nominal precedido de preposición $(20 \mathrm{~b}, 21,22,23)$.

Veamos ahora qué sucede con la variación de la estructura lexical y aspecto morfológico en este tipo de construcciones.

24a) Se dictaron clases de dactilografia. (Barrenechea-Rosetti, 1979: 62) PRET. PERF. s. /3pl. S (T)/ pl.

24b) Se dictan/dictaban clases de dactilografia. Proceso Durativo/No puntual

PTE PRET.IMPERF/3pl S (P)/pl.

24c) Se dicta/ dictaba una clase de dactilografia por semana en ese instituto. PTE/3sg PRET.IMPERF/3sg S (T/P)/sg. CIRC (Tpo-FREC) CIRC (Lug)

24d) Las clases de dactilografia se dictan normalmente.
$\mathrm{S}(\mathrm{T}) / \mathrm{pl}$.
PTE/3pl CIRC (Tpo)

24e) María (?) dicta/dictaba clases de dactilografia durante el verano. (VA- TR) $S$ (A) PTE/PRET.IMPERF/3sg O(T)/pl. CIRC

24f) Las clases de dactilografia, ese verano, fueron dictadas por un alumno avanzado.

$\mathrm{S}(\mathrm{T}) / \mathrm{pl}$. CIRC FV. PASIVA / 3pl Complemento Agente/sg

PRET.PERF.s+PPIO PDO (VP.Perifrástica)

Si tenemos en cuenta, como lo sugiere Maldonado, la estructura o aspecto lexical, el verbo dictar puede ser considerado según su significado inherente como un predicado estable, una actividad ${ }^{83}$, sin demarcación temporal (atélico, durativo) y agentivo en tanto se puede identificar a un agente con control, volición, responsable de ejecutar la actividad.

Al observar el aspecto morfológico, notamos que:

- la marca morfológica perfectiva en (24a) aporta telicidad o demarcación temporal. En la combinación Predicado estable (actividad) + marca perfectiva (pretérito perfecto simple) cambian las propiedades aspectuales del predicado por el aspecto morfológico $\mathrm{y}$, en consecuencia, el evento es visto como actividad concluida, realización ${ }^{84}$ (predicado cambiante).

- la marcación imperfectiva, en cambio, propicia una lectura habitual o genérica. En la combinación Predicado estable + marca imperfectiva vemos una lectura habitual en (24b),

\footnotetext{
${ }^{83}$ Maldonado (1999: 294), siguiendo a Vendler (1967), define las actividades como procesos homogéneos para los que cada parte del proceso es de la misma naturaleza que la totalidad del evento; por ej.: caminar.

${ }^{84}$ Las realizaciones (accomplishments): son definidas como cambios que de manera natural presuponen duración: Ayer escribió la carta; El año pasado construyó dos casas. (Ibídem, 294).
} 
rutinaria en (24d), iterativa en (24c) o genérica (24b). Todas estas lecturas focalizan el evento como un proceso y, en combinación con giros adverbiales temporales o tiempos verbales, subrayan distintos momentos de ese proceso. Estas lecturas se oponen a la focalización del evento como un todo acabado, rasgo típico de las construcciones MPT vistas anteriormente.

Finalmente, cabe seffalar un último rasgo del aspecto lexical: su grado de agentividad. A diferencia de verbos como perderse, romperse, quebrarse o tildarse, dictar presupone un agente con control y volición de sus actos: un agente humano.

Este último criterio nos permite conceptualizar un continuum de eventos o tipos de situaciones en las que, a primera vista, se observa $+/$ - transitividad, según podamos distinguir un participante (Sujeto Agente) que inicia deliberadamente una acción que afecta a otro participante (P). En la tabla 2 mencionamos sintéticamente los tipos de construcciones observadas en el trabajo, ordenadas según una escala de agentividad creciente.

\begin{tabular}{|l|l|l|}
\hline \multicolumn{2}{|c|}{ Tipo de construcción } & Ejemplo \\
\hline E & Media Espontánea -Cambio de & La taza se rompió. / El agua se volcó. \\
S & estado o posición del P/T- & La planta se quebró. \\
T & R & \\
\cline { 2 - 3 } U & Medio-pasiva & Estos libros se venden bien. \\
C & -imperfectiva/genérica; & \\
T & cualidad inherente al S (P/T) - & \\
\cline { 2 - 3 } R & Pasiva -perfectiva/ & El edificio se construyó en 1963. \\
A & Promocional- & Muchos libros se repartieron. \\
\cline { 2 - 3 } S A +CAU & \\
C & Impersonal -imperfectiva- & Se ha dicho la verdad. \\
O & Promocional & Se aprueba la ley. \\
N & & \\
\cline { 2 - 3 } S & Impersonal -perfectiva/ No & Se honró a los héroes de la patria. \\
E & promocional- & Ya se ha avisado a los bomberos. \\
\hline
\end{tabular}

TABLA 2. CONSTRUCCIONES CON SE: MEDIA, PASIVA E IMPERSONAL. (ADAPTADO DE MALDONADO, 1999; MENDIKOETXEA, 1999)

En la tabla 3 figuran los rasgos semántico-sintácticos y pragmáticos que caracterizan los tipos de estructuras observadas. 


\begin{tabular}{|c|c|c|c|c|}
\hline \begin{tabular}{l|l} 
TIPO & DE \\
CONSTRUCCIÓN
\end{tabular} & $\begin{array}{l}\text { TIPO DE } \\
\text { SITUACIÓN - } \\
\text { VERBO }\end{array}$ & $\begin{array}{l}\text { RASGOS SEMÁNTICO- } \\
\text { PRAGMÁTICOS del } \\
\text { argumento S }\end{array}$ & $\begin{array}{l}\text { RASGOS } \\
\text { SEMÁNTICO- } \\
\text { PRAGMÁTI- } \\
\text { COS del } \\
\text { argumento o }\end{array}$ & Estatus del SE \\
\hline $\begin{array}{l}\text { Media Espontánea o } \\
\text { decausativa }\end{array}$ & $\begin{array}{l}\text { Evento } \\
\text { Cambio de } \\
\text { estado o posición } \\
\text { de P/T. } \\
\text { Perfectivo. }\end{array}$ & $\begin{array}{l}\text { Paciente: } \\
\text { Afectado, Individualizado, } \\
\pm \text { Animado. } \\
\text { Tópico (concordante; } \\
\text { posverbal) }\end{array}$ & מח- & $\begin{array}{lr}\text { Afijo } & \text { verbal. } \\
\text { Focaliza } & \text { el } \\
\text { cambio } & - \text { sin } \\
\text { causación externa- } \\
\text { del argumento } \\
\text { afectado (P). }\end{array}$ \\
\hline Medio-pasiva- & $\begin{array}{l}\text { Evento: predica } \\
\text { una cualidad } \\
\text { inherente a } \\
\mathrm{S}(\mathrm{P} / \mathrm{T}) \text { - } \\
\text { Imperfectivo }\end{array}$ & $\begin{array}{l}\text { Tema: } \\
\text { Afectado, Individualizado, } \\
\pm \text { Animado. } \\
\text { Tópico (concordante; } \\
\text { posverbal) }\end{array}$ & ב-ב- & $\begin{array}{l}\text { Afijo verbal. } \\
\text { Lectura genérica. }\end{array}$ \\
\hline Pasiva Promocional & $\begin{array}{l}\text { Evento - } \\
\text { perfectivo }\end{array}$ & $\begin{array}{l}\text { Paciente/Tema: } \\
\text { Afectado/Efectuado } \\
\text { Individualizado, } \\
\pm \text { Animado. } \\
\text { T6́pico (concordante) } \\
\text { Pre/ Posverbal }\end{array}$ & & $\begin{array}{l}\text { Afijo verbal } \\
\text { Focaliza el evento } \\
\text { concluido, estado } \\
\text { resultante. }\end{array}$ \\
\hline $\begin{array}{l}\text { Impersonal - } \\
\text { Promocional }\end{array}$ & $\begin{array}{l}\text { Evento- } \\
\text { Imperfectivo }\end{array}$ & $\begin{array}{l}\text { Agente Humano } \\
\text { lexicalizado en el verbo. } \\
\text { Paciente/Tema: } \\
\text { Afectado/Efectuado } \\
\text { Individualizado. } \\
\text { Topicalizado. }\end{array}$ & - & $\begin{array}{l}\text { Afijo verbal. } \\
\text { Lectura } \\
\text { impersonal, i.e. } \\
\text { con agente no } \\
\text { focalizado/individ } \\
\text { ualizado } \\
\text { (genérica, } \\
\text { habitual, } \\
\text { universal) }\end{array}$ \\
\hline $\begin{array}{l}\text { Impersonal - No } \\
\text { promocional- }\end{array}$ & $\begin{array}{l}\text { Evento- } \\
\text { Actividad. } \\
\text { Perfectiva }\end{array}$ & $\begin{array}{l}\text { Agente humano } \\
\text { lexicalizado en la base } \\
\text { verbal. } \\
\pm \text { Tópico. }\end{array}$ & $\begin{array}{l}\text { Paciente/ } \\
\text { Tema: } \\
\text { Afectado o } \\
\text { Efectuado. } \\
\text { Individualizado } \\
\text { Relevante. }\end{array}$ & $\begin{array}{l}\text { Afijo verbal. } \\
\text { Lectura } \\
\text { impersonal con } \\
\text { agente no } \\
\text { focalizado/individ } \\
\text { ualizado } \\
\text { (genérica, } \\
\text { habitual, } \\
\text { universal) } \\
\end{array}$ \\
\hline
\end{tabular}

TABLA 3: RASGOS SINTÁCTICO-SEMANTICO-PRAGMÄTICOS DE LAS CONSTRUCCIONES CON SE: MEDIO-PASIVAS E IMPERSONALES.

\section{Consideraciones finales}

En este trabajo hemos intentado caracterizar $y$ definir las construcciones tradicionalmente denominadas pasivas e impersonales, combinando dos aportes explicativos: la linguística estructural y la tipología funcional. La primera nos permitió relevar las diferencias formales que oponen pasivas (verbo activo + SNplural= Sujeto), impersonales (verbo activo + SN precedido de preposición $=$ Objeto) y pasiva perifrástica $($ SNS preverbal + verbo pasivo 
(complemento agente optativo). Observamos que el criterio de la concordancia no resulta suficiente para definir la lectura pasiva o impersonal de un enunciado como $\mathrm{Ya}$ se resolvieron esos problemas/ya se resolvió ese problema. Para desambiguar vimos que era necesario considerar además, aspectos semánticos y pragmáticos.

En este sentido, la lingúística tipológica y funcional nos ofrece una tipología de operaciones de ajuste de valencia y oposiciones de voz que nos permite comprender y explicar los cambios introducidos por el clítico se.

Si bien los términos empleados en los trabajos descriptivos y gramáticas del espafiol son muy diferentes, podemos distinguir básicamente tres tipos de estructuras destransitivizadas (media espontánea, pasiva e impersonal) que comparten además de la presencia obligatoria del clítico etimológicamente reflexivo, el verbo intransitivizado y un único sintagma nominal. Estas estructuras pueden ser agrupadas en un continuum \pm volitivo; \pm agentivo/Causativo. En uno de los extremos podemos ubicar las construcciones espontáneas en las que el participante afectado entra en el cambio de estado sin una causación externa explícita o conocida como en Se rompió la taza. En el otro extremo del continuum podemos ubicar aquellas construcciones que poseen lexicalizado en el verbo al inductor o agente del evento, aunque por motivos semánticopragmáticos no está explicito en el enunciado como por ejemplo en No se ha dicho toda la verdad. En todo el subsistema medio-pasivo-impersonal entran en juego numerosos aspectos semántico-pragmáticos por ello, creemos que es necesario atender la estructura léxicosemántica del verbo, los rasgos semántico-sintácticos de los argumentos nominales así como los efectos pragmáticos buscados, para comprender la relación entre estas estructuras.

\section{Referencias bibliográficas}

BARRENECHEA, A. M. - MANACORDA DE ROSETTI, M. (1984) Estudios de gramática estructural. Bs. As., Paidós.

BARRENECHEA, A. M. - MANACORDA DE ROSETTI y otros. (1979) La voz pasiva en el español hablado en Buenos Aires. En: Estudios lingüisticos y dialectológicos. Temas hispanos. Bs. As., Ed Hachette.

BOGARD, S. (2005) Antipasivas en español. Forma y función. Ponencia para el TERCER SEMINARIO DE VOZ, CAMBIO DE VALENCIA Y FORMACIÓN DE PALABRA. México, Universidad de Sonora.

BOGARD, S. (2008) Los clíticos pronominales del español. Una visión de conjunto. Ponencia presentada en el VII Coloquio de Linguística en la ENAH (Escuela Nacional de Antropología e Historia). México. $37 \mathrm{p}$.

DI TULIO, A. (2005) Manual de gramática del español. Bs. As, La isla de la Luna. 
GARCIA-MIGUEL, J. M. (2001a) Algunas motivaciones en las tipologias de las variaciones de diátesis: Sistemas actanciales y polisemia de los morfemas de voz. En: Silva, A (org): Linguagem e Cognição: A Perspectiva da lingúistica cognitiva. APL/UCP Braga.

(2001b). Tipología de las variaciones de diátesis en lenguas amerindias. En: Calvo, Julio (Ed) Contacto interlinguístico e intercultural en el mundo hispano. Vol. I: 209238. Valencia, Universitat de Valencia (IVALCA).

GIVÓN, T. (1984) Syntax. A functional-typological introduction. Vol. . I. Amsterdam/Philadelphia: John Benjamins Publishing Co.

HOPPER, P y THOMPSON, S. (1980) Transitivity in grammar and discourse.Language 56. P 251-299.

MALDONADO, R. (1999) A media voz. Problemas conceptuales del clítico se. México, UNAM.

MENDIKOETXEA, A.(1999) Construcciones inacusativas y pasivas. En Bosque, I y Demonte, V., dirs.: Gramática descriptiva de la lengua española. Vol 2. Las construccciones sintácticas fundamentales -Relaciones temporales, aspectuales y modales. Madrid, Espasa Calpe. Pág.1575-1629

MENDIKOETXEA, A.(1999) Construcciones con se: medias, pasivas e impersonales.En Bosque, I y Demonte, V., dirs.: Gramática descriptiva de la lengua española. Vol 2. Las construccciones sintácticas fundamentales -Relaciones temporales, aspectuales y modales. Madrid, Espasa Calpe. Pág.1631-1722.

PAYNE, T. (1997). Describing morphosyntax. A guide for field linguists. New York: Cambridge University Press.

\section{Breve nota biográfica de la autora}

Adriana Zurlo es Profesora de Lengua y Literatura (2005) y Lic.en Letras (2010), egresada de la UNNE. Actualmente se desempeña en el NELMA (IIGHI. Conicet) como becaria de Posgrado Tipo I con el proyecto titulado Sistema medio en dos lenguas de Resistencia: español y toba. Estudio tipológico funcional y cognitivo, que también constituye el proyecto de tesis doctoral, ambos bajo la dirección del Dr. Sergio Bogard Sierra y la Dra. M. Censabella. Ha colaborado, como adscripta egresada en la cátedra Linguística I (2007) y en Historia del Español (2009-2010). 